\title{
Temperature structure of the intergalactic medium within seven nearby and bright clusters of galaxies observed with XMM-Newton
}

\author{
H. Bourdin ${ }^{1}$ and P. Mazzotta ${ }^{1,2}$ \\ 1 Dipartimento di Fisica, Università degli Studi di Roma "Tor Vergata”, via della Ricerca Scientifica, 1, 00133 Roma, Italy \\ e-mail: [herve.bourdin; pasquale.mazzotta] @roma2.infn.it \\ 2 Harvard-Smithsonian Center for Astrophysics, 60 Garden Street, Cambridge, MA 02138, USA
}

Received 5 June 2006 / Accepted 31 October 2007

ABSTRACT

\begin{abstract}
Aims. Using a newly developed algorithm, we map, to the highest angular resolution allowed by the data, the temperature structure of the intra-cluster medium (ICM) within a nearly complete X-ray flux limited sample of galaxy clusters in the redshift range between $z=0.045$ and $z=0.096$. Our sample contains seven bright clusters of galaxies observed with XMM-Newton: Abell 399, Abell 401, Abell 478, Abell 1795, Abell 2029, Abell 2065, Abell 2256.

Methods. We use a multi-scale spectral mapping algorithm especially designed to map spectroscopic observables from X-ray extended emission of the ICM. By means of a wavelet analysis, this algorithm couples spatially resolved spectroscopy with a structure detection approach. Derived from a former algorithm using Haar wavelets, our algorithm is now implemented with B-spline wavelets in order to perform a more regular analysis of the signal. Compared to other adaptive algorithms, our method has the advantage of analysing spatially the gas temperature structure itself, instead of being primarily driven by the geometry of gas brightness.

Results. For the four clusters in our sample that are major mergers, we find a rather complex thermal structure with strong thermal variations consistent with their dynamics. For two of them, A2065 and A2256, we perform a 3-d analysis of cold front-like features evidenced from the gas temperature and brightness maps. Furthermore, we detect a significant non-radial thermal structure outside the cool core region of the other 3 more "regular" clusters, with relative amplitudes of about about $10 \%$ and typical sizes ranging between 2 and 3 arcmin. We investigate possible implications of this thermal structure on the mass estimates, by extracting the surface brightness and temperature profiles from complementary sectors in the "regular" clusters A1795 and A2029, corresponding to hottest and coldest regions in the maps. For A2029, the temperature and surface brightness gradients seem to compensate each other, leading to a consistent mass profile. For A1795, however, the temperature structure leads to a significant mass discrepancy in the innermost cluster region. The third "regular" cluster, A478, is located in a particular sky region characterised by strong variations of neutral hydrogen column density, Nh, even on angular scales smaller than the cluster itself. For this cluster, we derive a spectroscopic $\mathrm{Nh}$ map and investigate the origin of Nh structure by discussing its correlation with galactic emission of dust in the infrared.
\end{abstract}

Key words. galaxies: clusters: general - galaxies: intergalactic medium - X-rays: galaxies: clusters techniques: image processing - techniques: spectroscopic

\section{Introduction}

Clusters of galaxies are thought to form by accretion of less massive groups and clusters under the influence of gravity. Following this scheme, they successively overcome some transient merging processes that alter their energy content and some relaxation phases, which lead to the almost virialised systems we observe.

The thermodynamical state of X-ray emitting intra-cluster medium (ICM) depends on both the cluster merger history and some not yet perfectly understood physical processes that drives its thermalisation, such as heat conduction or viscosity. Revealed by X-ray observations, the brightness and temperature structure of the ICM testifies to this final state. The brightness structure of the ICM has been extensively studied in the past (e.g. Forman \& Jones 1982; Slezak et al. 1994; Buote \& Tsai 1996; Schuecker et al. 2001). The current generation X-ray telescopes (XMM-Newton, Chandra) now further allow us to accurately map and investigate the temperature structure of the ICM (see e.g. Bauer et al. 2005), provided that specific algorithms help at optimising a necessary compromise between the precision of local temperature measurements and the spatial resolution of temperature maps.
For this reason, a number of spectral-mapping algorithms have been recently developed in X-ray astronomy. All of them can be essentially subdivided in three categories, depending on the general approach used. A first approach combines X-ray imaging in several energy bands, and colour analyses obtained from recombination of multi-band information. The imaging part of these algorithms usually requires de-noising by adaptive smoothing (e.g. Markevitch et al. 2000). A second approach proposes performing spatially resolved spectroscopy with required signal-to-noise. To do so, the field of view is firstly sampled in independent spatial bins following a mesh refinement scheme, then spectroscopic estimations are performed within each bin. Several binning strategies have been proposed in this context: adaptive binning (e.g. Sanders \& Fabian 2001), "contour binning" (Sanders 2006), or Voronoi tessellation (e.g. Cappellari \& Copin 2003). Proposed by Bourdin et al. (2004), a third approach consists in using Haar wavelet coefficients in order to couple a multi-scale spectroscopic analysis with a structure detection scheme.

Among these algorithms, only the last one performs a direct investigation of structure for the searched parameter itself - i.e. the ICM projected temperature - while the signal analysis 
Table 1. Cluster sample with associated redshift, coordinates, total ICM flux from the BCS survey (Ebeling et al. 1998), and P4/P0 power ratio reported in Buote \& Tsai (1996). Last column: average neutral hydrogen column density measured along line of sight of each cluster (Dickey \& Lockman 1990).

\begin{tabular}{cccccc}
\hline $\begin{array}{c}\text { Cluster } \\
\text { name }\end{array}$ & Redshift & $\begin{array}{c}\text { Equatorial coordinates } \\
\text { (J2000) }\end{array}$ & $\begin{array}{c}\text { ICM flux } \\
\left(10^{-15} \mathrm{~W} \mathrm{~m}^{-2} / 10^{-12} \mathrm{erg} \mathrm{s}^{-1} \mathrm{~cm}^{-2}\right)\end{array}$ & $\begin{array}{c}\text { Power ratio } \\
P 4 / P 0\end{array}$ & $\begin{array}{c}\text { Neutral hydrogen column } \\
\text { density }\left(10^{24} \mathrm{~m}^{-2}\right)\end{array}$ \\
\hline & & Relaxed clusters & \\
A478 & 0.088 & $041325.0+102754.0$ & 39.9 & 0.025 & 15.1 \\
A1795 & 0.062 & $134853.0+263532.0$ & 68.1 & 0.004 & 1.2 \\
A2029 & 0.077 & $151056.0+054442.0$ & 61.3 & 0.050 & 3.14 \\
\hline & & & & 10.9 \\
A399 & 0.072 & $025753.0+130200.0$ & Major merger clusters & - \\
A401 & 0.074 & $025858.0+133400.0$ & 28.8 & 0.157 & 10.5 \\
A2065 & 0.072 & $152242.0+274300.0$ & 22.8 & - & 2.95 \\
A2256 & 0.058 & $170358.3+783831.0$ & 49.5 & 0.395 & 4.1 \\
\hline
\end{tabular}

Table 2. Effective exposure time of each EPIC XMM-Newton observation. In brackets: fraction of the useful exposure time after solar-flare "cleaning".

\begin{tabular}{cccc}
\hline \hline $\begin{array}{c}\text { Cluster } \\
\text { name }\end{array}$ & $\begin{array}{c}\text { MOS1 effective } \\
\text { exposure time }(\mathrm{ks})\end{array}$ & $\begin{array}{c}\text { MOS2 effective } \\
\text { exposure time }(\mathrm{ks})\end{array}$ & $\begin{array}{c}\text { PN effective } \\
\text { exposure time }(\mathrm{ks})\end{array}$ \\
\hline A399 & $8.3(58.6 \%)$ & $4.6(32.8 \%)$ & $4.2(45.2 \%)$ \\
A401 & $12.0(92.5 \%)$ & $10.0(77.4 \%)$ & $2.8(35.1 \%)$ \\
A478 & $30.3(44.2 \%)$ & $38.0(30.5 \%)$ & $47.0(43.6 \%)$ \\
A1795 & $22.5(45.8 \%)$ & $23.5(47.8 \%)$ & $19.3(45.5 \%)$ \\
A2029 & $6.5(37.5 \%)$ & $7.7(44.4 \%)$ & $6.4(50.6 \%)$ \\
A2065 & $20.4(61.0 \%)$ & $19.9(60.3 \%)$ & $12.2(55.8 \%)$ \\
\hline A2256(1) & $8.7(62.1 \%)$ & $8.5(60.3 \%)$ & $4.8(54.8 \%)$ \\
A2256 (2) & $7.9(48.5 \%)$ & $8.6(53.0 \%)$ & $5.9(50.9 \%)$ \\
A2256 (3) & $8.6(47.3 \%)$ & $8.9(48.6 \%)$ & $5.3(26.3 \%)$ \\
A2256 (4) & $7.8(47.8 \%)$ & $7.1(43.3 \%)$ & $6.5(58.0 \%)$ \\
\hline
\end{tabular}

is essentially driven by the geometry of gas brightness in all the other algorithms. Here, we present a new version of the algorithm of Bourdin et al. (2004), now implemented with B-spline wavelets and possibly enabling us to apply a more conservative thresholding strategy. Other improvements are related to adaptations to more recent calibration of XMM-Newton instruments, which particularly allow us to combine data coming from the three European Photon Imaging Cameras (EPIC).

This algorithm has been used to perform a systematic study of a nearly complete X-ray flux selected sample of seven clusters observed with XMM-Newton. Along this paper, we first discuss the sample selection and data preparation in Sects. 2 and 3 , then expose our data analysis scheme in Sect. 4, and finally give a brief description of the thermal structure observed in each single cluster in Sect. 5. Before providing general discussions and conclusions in Sect. 9, we discuss more specific issues related to thermal features observed within individual objects: isoradial thermal structure in the relaxed clusters Abell 1795 and Abell 2029 (Sect. 6), cold fronts in the merging clusters Abell 2065 and Abell 2256 (Sect. 7). The additional Sect. 8 is related to the high neutral hydrogen column density variations observed across the field of view of Abell 478.

The cluster radii are computed as angular diameter distances, assuming a $\lambda$-CDM cosmology with $H_{0}=70 \mathrm{~km} \mathrm{~s}^{-1} \mathrm{Mpc}^{-1}$, $\Omega_{0}=0.3, \Omega_{\Lambda}=0.7$.

\section{The cluster sample}

Starting from the X-ray Brightest Cluster Sample (BCS) of Ebeling et al. (1998), we selected a flux limited sample of clusters. In order for the clusters to have an observed angular size close to the EPIC cameras field of view, we limited the cluster redshift selection to the range $\delta z=[0.045,0.096]$, corresponding to an angular size from 5 to 10 arcmin.

Our selection criteria returned eight bright clusters, namely Abell 2142, Abell 2029, Abell 478, Abell 1795, Abell 401, Abell 2256, Abell 399, Abell 2065, public XMM-Newton data being available for each of them. However, since the Abell 2142 observation is strongly altered by solar flares, we decided to remove it from our sample and focus on the seven remaining clusters.

Previous investigations, based primarily on the analysis of the X-ray surface brightness, classified 4 of the objects in our sample as major mergers and the remaining 3 as more "relaxed" clusters. The clusters are listed in Table 1 and are grouped according to this classification. The cluster redshifts and the ICM brightness from the BCS survey are reported in the same table, with associated $\mathrm{P} 4 / \mathrm{P} 0$ power ratio calculated by Buote \& Tsai (1996) when available. Expected to be an indicator of the dynamical evolution stage of the cluster, this latter quantity is lower for the 3 most relaxed clusters than for the clusters classified as major mergers.

\section{Observations and data preparation}

\subsection{Observations}

All data used for this investigation come from the EPIC XMMNewton database. We use individual observations of Abell 399, Abell 401, Abell 478, Abell 1795, Abell 2029, and Abell 2065, and a multiple pointing observation of Abell 2256. For all of these clusters, data sets combine observations obtained from the 
three EPIC instruments: MOS1, MOS2 and PN. A summary of data sets is provided in Table 2, with associated exposure times.

\subsection{Data filtering}

In addition to the extended and optically thin emission of ICM, $\mathrm{X}$-ray observations gather photon impact events related to other sources, such as spatially resolved X-ray emitting galaxies and the cosmic X-ray background (CXB). Moreover, observations may be transiently contaminated by solar flares. While the extended contributions of ICM and CXB are hardly separable, contributions from point-sources and solar flares can be isolated and removed from the observed signal through spatial and temporal wavelet analyses, respectively.

In order to detect high solar flares periods and remove corresponding data sets, we analyse light curves with associated high energy events (10-12 keV) and softer events (1-5 keV), respectively. As proposed by Nevalainen et al. (2005), this two-step analysis first enables us to isolate the most prominent flares at high energy, where ICM brightness is expected to be negligible, then detect some additional contribution of soft flares only. For each of these curves, we use a B3-spline "à-trous" wavelet algorithm in order to detect disruptions, and select the positive irregularities with amplitude overcoming a $2 \sigma$ significance threshold with regard to the light-curve fluctuation. This "cleaning" process has significantly lowered the effective exposure time of observations, as reported for individual observations and pointings on Table 2.

In order to identify point-sources, we analyse EPIC-MOS event-lists which are more suited to imaging, and adopt an object separation algorithm derived from the multi-scale vision model of Bijaoui \& Rue (1995). After detecting and imaging pointsources, we associate a mask to regions of the field of view dominated by point-sources contribution, and isolate events coming from these regions when analysing the signal.

\subsection{Data sampling - effective exposure}

XMM-Newton imaging cameras provide photon impact event lists with associated energy and position on the detector planes. In order to analyse our signal using discrete wavelet algorithms, we group events coming from various observations, if any, and sample them spatially into sky coordinate grids, with given angular resolution, $a_{0}$. We also sample events in energy so as to perform spectral analyses, which leads to $3 \mathrm{D}$ event cubes associated with each EPIC instrument, and sampled in position $(k, l)$ and energy $(e)$.

For imaging and spectral-mapping purposes, we associate to these event cubes a set of local "effective exposure" $E(k, l, e)$ and "background" cubes, $n_{\text {bck }} F_{\text {bck }}(k, l, e)$, with similar positionenergy sampling, and use them for modelling the bolometric or spectroscopic ICM radiation; we report details about the background modelling in Appendix A. Let us define here the "effective exposure" $E(k, l, e)$ at pixel $[k, l]$ as the linear combination of CCD exposure times $t_{\mathrm{CCD}}(k, l, p)$ related to individual observations $p$, with correction for spatial variations of the mirror effective area or so-called "vignetting factor", $\Delta a_{\text {mirror }}(k, l, e)$, transmission by other focal instrument, - i.e. reflection grating spectrometer $(\mathrm{RGS})-t r_{\mathrm{RGS}}$, anddetector pixel area with correction for gaps and bad pixels, $a_{\mathrm{CCD}}(k, l)^{1}$. For K observations, we get:

$$
\begin{aligned}
E(k, l, e)= & \sum_{p=1}^{\mathrm{K}} t_{\mathrm{CCD}}(k, l, p) \times \Delta a_{\mathrm{mirror}}(k, l, e, p) \\
& \times \operatorname{tr}_{\mathrm{RGS}}(k, l, e, p) \times a_{\mathrm{CCD}}(k, l, p) .
\end{aligned}
$$

\section{Data analysis}

\subsection{ICM temperature mapping}

In order to map the ICM temperature structure, we have built a spectral mapping algorithm coupling a spectroscopic and multiscale analysis of the X-ray signal, to a wavelet mapping of the searched parameter structure.

Mainly following the scheme of the Bourdin et al. (2004) algorithm - hereafter the 2004 algorithm - we first sample the field of view using redundant and square grids with typical size $s_{j}=2^{j} a_{0},\left\{j \in\left[0, j_{\max }\right]\right\}$, according to a dyadic scheme. Then, we locally estimate the gas temperature $T$ and its fluctuation $\sigma_{T}$ by fitting a spectral model to the data, within each meta-pixel $[k, l, j]$ of the different grids. A set of temperature maps $T(k, l, j)$ is obtained, with associated noise expectation maps $\sigma_{T}(k, l, j)$. Filtering the $T(k, l, j)$ and $\sigma_{T}(k, l, j)$ maps using high-pass analysis filter enables us to code the temperature variations as wavelet coefficients $W_{T}(k, l, j)$ with expected noise $\sigma_{W_{T}}(k, l, j)$, and to detect significant temperature structures as wavelet coefficients with amplitude overcoming a significance threshold depending on $\sigma_{W_{T}}(k, l, j)$. Finally, we map the gas temperature using a Tikhonov regularised thresholding of the wavelet transform.

More detailed in Bourdin et al. (2004), this general approach has been adapted to the present study. In particular, the local spectral fitting is now implemented using an updated plasma emission code and now allows a multiple parameter estimation. Furthermore, a B-spline wavelet transform is now used instead of the Haar wavelet transform. Below we describe both of these improvements in detail.

\subsubsection{Spectral fitting of local ICM temperature}

The local estimation of ICM temperature is performed by fitting a normalised spectral model, $F_{\text {evt }}\left(T, Z, N_{\mathrm{h}}, e\right)$, to the data set associated with meta-pixel $[k, l, j]$. Combining contributions of ICM itself, $n_{\mathrm{ICM}}(k, l) F_{\mathrm{ICM}}\left(T, Z, N_{\mathrm{h}}, e\right)$, and "overall background", $n_{\mathrm{bck}} F_{\mathrm{bck}}(k, l, e)$, this model is sampled in photon energies, $e$, and depends on the ICM temperature $T$, metal abundances, $Z$, and neutral hydrogen density column along the line of sight, $N_{\mathrm{h}}$ :

$$
\begin{aligned}
& n_{\mathrm{evt}}(k, l) F_{\mathrm{evt}}\left(T, Z, N_{\mathrm{h}}, e\right)= \\
& \quad E(k, l, e) \times n_{\mathrm{ICM}}(k, l) F_{\mathrm{ICM}}\left(T, Z, N_{\mathrm{h}}, e\right)+n_{\mathrm{bck}} F_{\mathrm{bck}}(k, l, e) .
\end{aligned}
$$

The unnormalised ICM contribution $F_{\mathrm{ICM}}\left(T, Z, N_{\mathrm{h}}, e\right)$ is modelled from plasma radiation flux $\phi_{\mathrm{ICM}}(T, Z, e)$, following the Astrophysical Plasma Emission Code (APEC, Smith et al. 2001) as a function of the gas temperature $T$ and heavy elements abundances $Z$, being set according to the solar element composition of Grevesse \& Sauval (1998). It is obtained by red shifting and

\footnotetext{
1 Information about these instrumental effects are provided in the following XMM-Newton Current Calibration Files (CCF), corresponding to each observation epoch: RGS*_QUANTUMEF, XRT*_XAREAEF, EMOS*_LINCOORD, EPN_LINCOORD, EMOS*_BADPIX, EPN_BADDPIX.
} 
distorting $\phi(T, Z, e)$ in order to take into account X-ray absorption by the galactic neutral hydrogen $h\left(N_{\mathrm{h}}, e\right)$ with given column density along the line of sight, $N_{\mathrm{h}}$, following absorption parameters of Balucinska-Church \& McCammon (1992). Introducing the instrument area $A(e)$, and convolving ICM radiation flux by the detector response in energy $R(e)^{2}$, we get the radiation power measured by the instrument for a given emitting source at redshift $z$ :

$$
\begin{aligned}
F_{\mathrm{ICM}}\left(T, Z, N_{\mathrm{h}}, e\right)= & R(e) \#\left[A(e) \times h\left(N_{\mathrm{h}}, e\right)\right. \\
& \left.\times \frac{\phi_{\mathrm{ICM}}[T, Z,(1+z) e]}{1+z}\right],
\end{aligned}
$$

where instrument area $A(e)$ takes into account the effective area of mirrors $a_{\text {mirror }}(e)$, the filter transmission $\operatorname{tr}_{\text {filter }}(e)$ and detector quantum efficiency $q_{\mathrm{CCD}}(e)^{3}$ :

$A(e)=q_{\mathrm{CCD}}(e) \times t r_{\text {filter }}(e) \times a_{\text {mirror }}(e)$.

In order to get a robust estimation of ICM temperatures, whatever the local statistics are, the spectral fitting is performed by maximising the $\log$-likelihood function $\log L\left(T, Z, N_{\mathrm{h}}, e\right)=$ $\sum_{i} \log F_{\text {evt }}\left(T, e_{i}\right)$, where our spectral model is summed on all energy channels, $e_{i}$.

\subsubsection{Wavelet mapping of ICM temperature structure}

In order to map the ICM temperature structure at scale $j$, we analyse the spatial correlation of temperature measurements using wavelet coefficients $W_{T, j}(k, l)$, computed from the temperature maps $T_{j}(k, l)$. A trivial solution to this computation has been proposed in the 2004 algorithm: filtering the temperature maps $T_{j}(k, l)$ using Haar high-pass analysis filters enables one to get a set of Haar wavelet coefficients $W_{T, H, j}(k, l)$. Indeed, the Haar wavelet is the dual function of the top-hat smoothing kernel $\Pi_{j}(k, l)$, which may be applied to the searched map $T_{0}(k, l)$ for computing the maps $T_{j}(k, l)$, at scale $j$. However, thresholding Haar wavelet transforms usually generates square artifacts, in particular when analysing regular signals.

Due to the expected smoothness of our signal, a better analysis of its spatial correlations can be provided by the more regular B-spline wavelets; see Curry \& Schoenberg (1947) for definition and e.g. Mallat (1998) for application to wavelet bases. Indeed, we do not expect any strong discontinuities in our signal, due to joint effects of both the instrument PSF and the 2D projection of the gas temperature structure. Since B-spline wavelets are the dual functions of the $\mathrm{m}$ degree $\mathrm{B}$-spline interpolation functions obtained by $(m+1)$ self-convolving of a top-hat smoothing kernel, they can also be used in our context; starting from the temperature maps $T_{j}(k, l)$ and convolving them $(m)$ times by a tophat smoothing kernel $\Pi_{j}(k, l)$ provides a new set of smoothed maps $T_{S(m), j}(k, l)$, whose dual wavelet coefficients are $\mathrm{m}$ degree B-spline wavelet coefficients $W_{T, S(m), j}(k, l)$. Introducing the smoothing length $a=2^{j}$ at scale $j$, we get:

$$
\begin{aligned}
\Pi_{j}(k, l) & =\mathbf{1}_{\left[k-\frac{a}{2}, k+\frac{a}{2}-1\right]} \times \mathbf{1}_{\left[l-\frac{a}{2}, l+\frac{a}{2}-1\right]}, \\
T_{S(m)}(k, l, j) & =\Pi_{j}(k, l)^{(m)} \star T(k, l, j) .
\end{aligned}
$$

\footnotetext{
${ }^{2}$ Detector responses are tabulated within the XMM-Newton redistribution matrix files (RMF).

${ }^{3}$ Information about these instrumental effects are provided in the following XMM-Newton current calibration files (CCF), corresponding to each observation epoch: XRT*_XAREAEF, EMOS*_QUANTUMEF, EPN_QUANTUMEF, EMOS*_FILTERTRANSX, EPN_FILTERTRANSX.
}

Like the Haar wavelet, the B-spline wavelets can be projected in bi-orthogonal bases. We therefore adopted the shift-invariant Coifman \& Donoho (1995) algorithm for computing the wavelet coefficients $W_{T, S(m), j}(k, l)$, following a similar scheme as for the 2004 algorithm. To do so, the smoothed maps $T_{S(m)}(k, l, j)$ are convolved with a set of three high-pass analysis filters $h(k) g(l), g(k) h(l)$ and $g(k) g(l)$, associated with the B-spline wavelet, which leads to the three sets of wavelet coefficients $W_{T, S(m), j, h}(k, l), W_{T, S(m), j, v}(k, l)$ and $W_{T, S(m), j, d}(k, l)$ :

$W_{T, S(m), j, h}(k, l)=h(k) g(l) \star T_{S(m)}(k, l, j)$,

$W_{T, S(m), j, v}(k, l)=g(k) h(l) \star T_{S(m)}(k, l, j)$,

$W_{T, S(m), j, d}(k, l)=g(k) g(l) \star T_{S(m)}(k, l, j)$.

In order to re-construct the signal $T_{0}(k, l)$, the wavelet coefficients are first convolved using the high-pass inverse filters, $\tilde{h}(k) \tilde{g}(l), \tilde{g}(k) \tilde{h}(l)$ and $\tilde{g}(k) \tilde{g}(l)$ associated with the bi-orthogonal wavelet analysis and added to each other, which allows us to average the redundant information. Thus, the risk occurring when re-constructing the signal from the thresholded wavelet transform is significantly lowered.

Here we decided to work with the quadratic B-spline wavelet ( $m=2$, see Fig. 2), which is regular enough for our purpose but more compact than B-spline wavelets of higher degree. The quadratic B-spline analysis filters are reported in Table 3.

\subsection{ICM brightness mapping}

In order to map the ICM brightness structure, we have built an imaging algorithm where the local brightness $L_{\mathrm{X}}(k, l)$ is first estimated from a brightness model associated with pixel $[k, l]$, then iteratively de-noised in the scale-space by means of a discrete Haar wavelet analysis.

We estimate the local ICM brightness $L_{\mathrm{X}}(k, l)$ for energy band $\Delta e$, by correcting the number of events $n_{\mathrm{evt}}(k, l)$ detected at pixel $(k, l)$, from the expected "background" contribution, $n_{\text {bck }}(k, l)$, and exposure weighted instrument area, $a(k, l)$ :

$$
\begin{aligned}
a(k, l) & =\int_{\Delta e} E(k, l, e) F_{\mathrm{evt}}\left(T_{\mathrm{o}}, Z_{\mathrm{o}}, N_{\mathrm{ho}}, e\right) \mathrm{d} e, \\
\widehat{L_{\mathrm{X}}}(k, l) & =\frac{n_{\mathrm{evt}}(k, l)-n_{\mathrm{bck}}(k, l)}{a(k, l)},
\end{aligned}
$$

where the weighted instrument area $a(k, l)$ is computed for a fixed ICM emission model, $F_{\text {evt }}\left(T_{\mathrm{o}}, Z_{\mathrm{o}}, N_{\mathrm{ho}}, e\right)$. "Corrected" photon maps obtained from local estimator $\widehat{L_{X}}(k, l)$ and for energy band $0.5-2.5 \mathrm{keV}$ are shown in Fig. 1.

The signal de-noising is then performed by thresholding Haar wavelet coefficients $W_{\widehat{L_{X}}}[k, l]$, associated with a multiscale analysis of $\widehat{L_{X}}(k, l)$ using redundant grids. Driven by a significance criterion of detected structure, this thresholding is set after estimating the probability density function (PDF) of noise wavelet coefficients, $P\left(W_{\widehat{L_{X}}}[k, l]\right)$. To do so, we deduce $P\left(W_{\widehat{L X}}[k, l]\right)$ from the PDF of noise wavelet coefficients associated with local event counts, $n_{\mathrm{evt}}$ :

$P\left(W_{\widehat{L_{\mathrm{X}}}}[k, l]\right)=\frac{P\left(W_{n_{\mathrm{ev}}}[k, l]\right)}{a[k, l]}$,

which enables us to use an analytical form of $P\left(W_{n_{\text {evt }}}[k, l]\right)$, first proposed by Bijaoui \& Jammal (2001) for Haar wavelet analyses of uniform Poisson noise. Introducing the modified Bessel function of order $m, I_{m}\left(n_{\mathrm{evt}}\right)$, we get:

$P\left(W_{n_{\mathrm{evt}}}\right)=\sum_{m=-\infty}^{\infty} \mathrm{e}^{-n_{\mathrm{evt}}} I_{m}\left(n_{\mathrm{evt}}\right) \delta\left(W_{n_{\mathrm{evt}}}-m\right)$. 

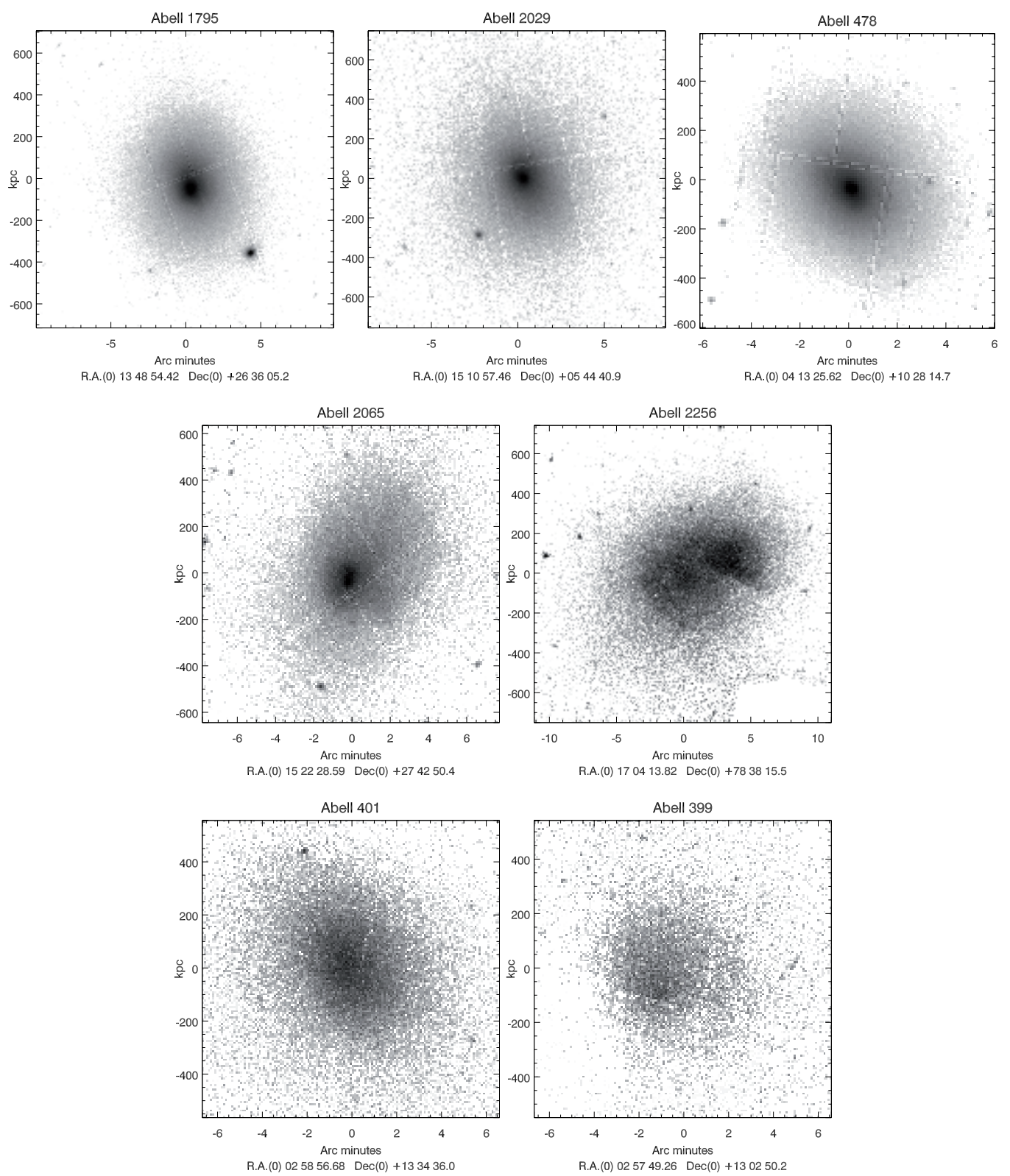

Fig. 1. EPIC-XMM "soft" (0.5-2.5 keV) exposures images of the clusters in our sample.

Table 3. The quadratic B-spline analysis filters.

\begin{tabular}{ccccccc}
\hline \hline & -2 & -1 & 0 & 1 & 2 & 3 \\
\hline$h$ & & .125 & .375 & .375 & .125 & \\
$g$ & & & -.5 & .5 & & \\
$\tilde{h}$ & & .125 & .375 & .375 & .125 & \\
$\tilde{g}$ & -.03125 & -.21875 & -.6875 & .6875 & .21875 & .03125 \\
\hline
\end{tabular}

\section{2D temperature structure}

The ICM temperature maps of each cluster in our sample are presented in Fig. 3, overlaid to the relative $0.3-2.5 \mathrm{keV}$ brightness isocontours levels obtained by wavelet imaging (see details above). The emission model $F_{\text {evt }}\left(T_{\mathrm{o}}, Z_{\mathrm{o}}, N_{\mathrm{ho}}, e\right)$ assumed for computing brightness maps has been obtained by spectral-fitting of an overall cluster spectrum excluding the core region of relaxed clusters.

The temperature maps have been computed from spectralfitting within the .7-7.5 keV energy band with fixed values of the
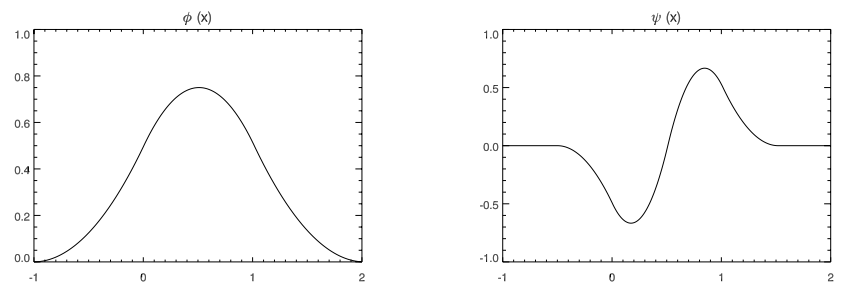

Fig. 2. The quadratic B-spline scaling function $\phi(x)$ and dual wavelet $\psi(x)$ in $1 \mathrm{D}$.

redshift, metal abundances, and neutral hydrogen column densities. The redshift values have been set to those in Table 1, while the metal abundances $Z_{\mathrm{o}}$ have been deduced from the spectral fitting of $F_{\text {evt }}\left(T_{\mathrm{o}}, Z_{\mathrm{o}}, N_{\mathrm{ho}}, e\right)$. For each cluster except Abell 478, the neutral hydrogen column densities, $N_{\mathrm{h}_{\mathrm{o}}}$, have been estimated by spectral fitting, similarly to the metal abundances. The values obtained are consistent with measurements by 

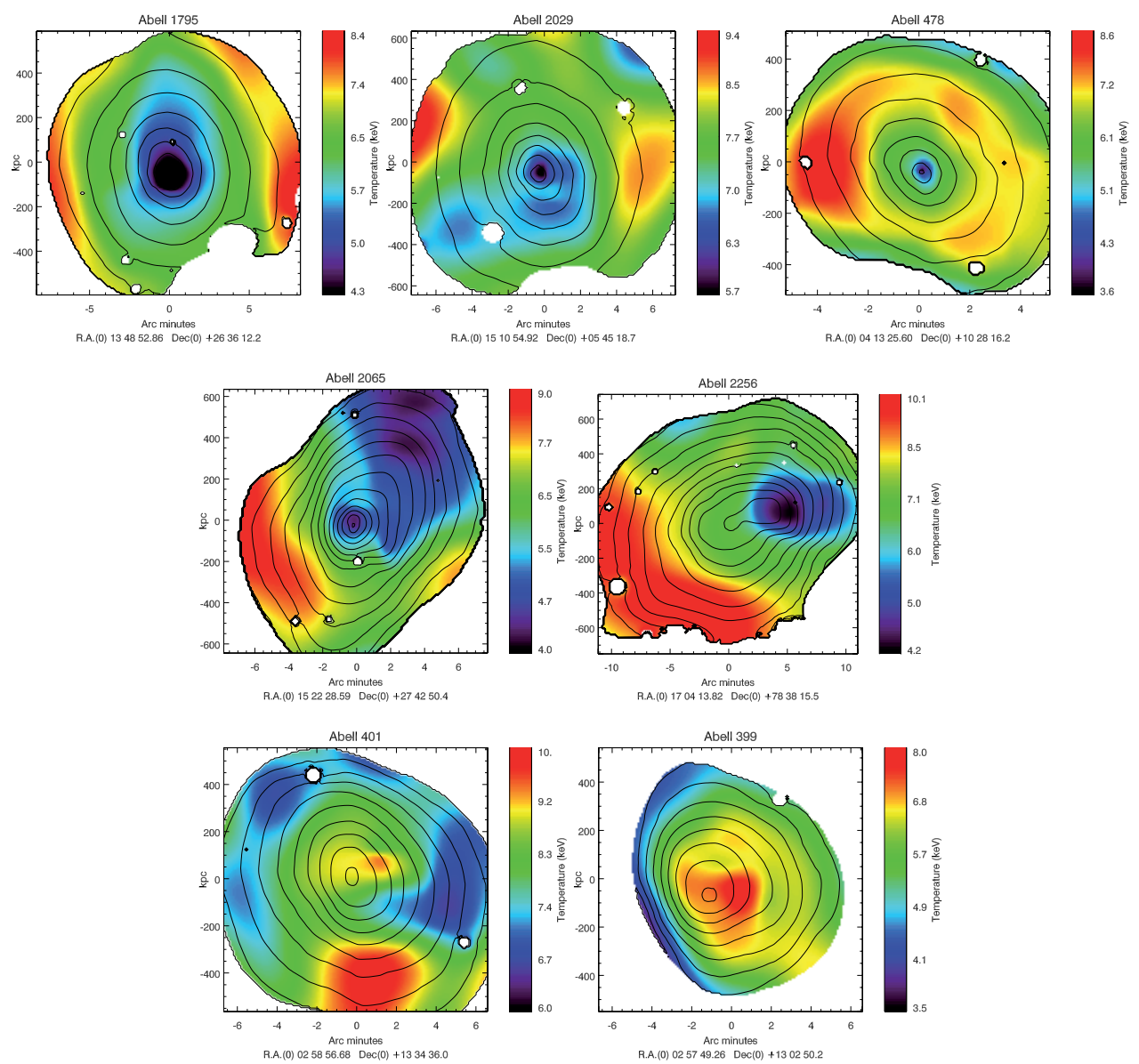

Fig. 3. ICM temperature maps of the clusters in our sample overlaid to the soft $(0.5-2.5 \mathrm{keV}) \mathrm{X}$-ray brightness isocontours. The isocontour levels are logarithmically equispaced by a factor $\sqrt{2}$.

Dickey \& Lockman (1990), in the 1 degree neighbourhood of each cluster centre (see Table 1). For A478, the $N_{\mathrm{h}}$ has been left as free parameter, since Dickey \& Lockman (1990) measurements were inconsistent with X-ray spectroscopic estimations (see Sect. 8 for details).

The EPIC-XMM field of view has been sampled in $256 \times$ 256 pixels, and the wavelet analysis has been unfolded over six spatial resolutions, allowing detection of features with typical size ranging from about 12 arcsec to $3.5 \mathrm{arcmin}$. The thresholding of the wavelet coefficients has been performed following the Donoho (1995) thresholding approach, leading to threshold levels that are always higher than the noise fluctuation $\sigma$ (typically from 1.75 to $2.5 \sigma$ with increasing analysis scales, thresholds lower than $2 \sigma$ being related to the highest resolution details, namely the cool core regions of relaxed clusters only).

In the following we give a short description of each cluster separately. For convenience we group the clusters following the dynamical classification, used in previous work and based on the $\mathrm{X}$-ray morphology, of relaxed and merging systems.

\subsection{Relaxed clusters}

\subsubsection{Abell 1795}

As observed in X-rays, Abell 1795 is known as an elliptical and relaxed cluster, as predicted by the relatively low $P 4 / P 0$ power ratios (see Table 1). The XMM-Newton observation of Abell 1795 confirms a globally elliptical symmetry for this cluster. Nevertheless, the XMM-Newton X-ray image in Fig. 1 shows the presence of a sharp surface brightness variation, at about 1 arcmin to the south with respect to the cluster centre. From a Chandra observation, this sharp surface brightness feature has been identified as a cold front and interpreted as the result of the sloshing of core gas within the cluster potential well (Markevitch et al. 2001). This scenario is consistent with what can be observed on surface brightness contours in Fig. 3, revealing at the same time a strong compression of isophotal lines across the cold front, and a shift of the cluster brightness peak towards the south, with regard to large radii isophotes.

The temperature map of Abell 1795 in Fig. 3 shows a global elliptical symmetry with a cool core. If we perform a radial analysis, it is consistent with temperature profiles derived from XMM-Newton (Tamura et al. 2001; Arnaud et al. 2001; Ikebe et al. 2004) and Chandra observations (Ettori et al. 2002; Markevitch et al. 2001; Vikhlinin et al. 2005). We notice, however, some anisotropies with regard to the overall elliptical symmetry of temperature structure. First of all, the cool core appears as being shifted towards the south, as coinciding with the cluster brightness peak. At larger radii $(r>2 \mathrm{arcmin})$ the cluster also presents some significant non radial thermal structure. In particular we find that the gas temperature is colder in a small sector to the north $(k T \simeq 5 \mathrm{keV})$ and hotter elsewhere $(k T \simeq 6 \mathrm{keV})$.

\subsubsection{Abell 2029}

Similarly to Abell 1795, Abell 2029 was also known as a regular and relaxed cluster (see also the $P 4 / P 0$ power ratios in 
Table 1). Except for its very central region where X-ray filaments have been observed (e.g. Clarke et al. 2004), no brightness nor temperature anisotropies have been previously detected for this cluster.

As already noticed by Vikhlinin et al. (2005), this cluster is projected near the galactic region of North Polar Spur, the X-ray spectral contribution of which has been modelled and added to the background model of Eq. (A.1). This contribution has been fitted simultaneously with cosmic background, in the same external region of the field-of-view, which led to a two thermal component model $\left(k T 1=0.22, k T 2=0.49, n_{K 1} / n_{J 2}=2.05\right)$, consistent with the analysis of Vikhlinin et al. $(2005)^{4}$.

The XMM-Newton image in Fig. 1 confirms that Abell 2029 is globally regular and elliptically symmetric (see also Fig. 3). The gas temperature map in Fig. 3 shows the presence of a cooler core $(k T \simeq 5.5 \mathrm{keV})$ and a positive radial temperature gradient that extends up to 1.5 arcmin from the cluster centre. If we perform a radial analysis, the temperature map is consistent with previously published temperature profiles (Lewis et al. 2003; Vikhlinin et al. 2005), except in the very central region $(r<10$ arcsec), where the Chandra data analysis of Lewis et al. (2003) indicates lower temperatures decreasing to about $2 \mathrm{keV}$.

Nevertheless, as observed for Abell 1795, even if the temperature structure is elliptically symmetric in the innermost regions, at larger radii the temperature map reveals some non radial thermal features with typical temperature variations of $\simeq 1 \mathrm{keV}$. In particular, we detect a cold region at 5 arcmin to the southeast of the cluster centre $(k T \simeq 6 \mathrm{keV})$.

\subsubsection{Abell 478}

Known to be regular and relaxed, Abell 478 has been observed recently by the Chandra and XMM-Newton telescopes (Sun et al. 2003; Pointecouteau et al. 2004). While the Chandra observation has revealed brightness substructure in the very centre of the cluster (Sun et al. 2003), both of these observations have shown a regular structure at larger radii. The elliptical symmetry and regularity of the surface brightness of Abell 478 is also evident from the X-ray cluster image in Fig. 1 and confirmed by the low value of the $P 4 / P 0$ power ratios in Table 1 .

The gas temperature map of Abell 478 is shown in Fig. 3. Due to the strong hydrogen column density appearing across the field of view $\left(N_{\mathrm{h}}=15.1 \times 10^{24} \mathrm{~m}^{-2}\right)$, the gas temperature map of this cluster has been computed from a spectral fitting process with a free $N_{\mathrm{h}}$ parameter. Using this procedure the temperature map appears remarkably regular with elliptical symmetry.

This map reveals a cool core $(k T \simeq 4 \mathrm{keV})$ with 1 arcmin radius, a radial temperature increase up to a plateau at about 3.5 arcmin from the cluster centre $(k T \simeq 7.5 \mathrm{keV})$, then a decrease up to 5 arcmin. This overall structure is in agreement with the shape of Chandra temperature profile published by Vikhlinin et al. (2005). Nevertheless, the absolute temperature values from our temperature map are lower than the values of Vikhlinin et al. (2005), and more consistent with the analysis of Pointecouteau et al. (2004), using the same data as here. Vikhlinin et al. (2005) and Sanderson et al. (2005) already noticed these discrepancies and argued that they are probably related to a combined effect of differences between instrument PSFs, and the complex temperature structure of the very central region in this cluster.

\footnotetext{
${ }^{4}$ Normalisation discrepancies with Vikhlinin et al. (2005) are due to different modellings of the CXB, our model already including a thermal component at $0.204 \mathrm{keV}$ (see Eq. (A.1)).
}

From both the XMM-Newton brightness and temperature maps we may conclude that Abell 478 is the most regular of our sample. Nevertheless, we should note that this cluster is located in a particular region of the sky characterised by strong variations of neutral hydrogen column density, on angular scales smaller than the cluster size itself. In this condition, the gas temperature variations are more difficult to detect for this cluster than for the other clusters of our sample (see also Sect. 8 below).

\subsection{Merging clusters}

\subsubsection{Abell $399-$ Abell 401}

Although, independently selected in our cluster sample, Abell 399 and Abell 401 form a close pair separated, in projected distance, by $3 \mathrm{Mpc}$ (36 arcmin). The brightness contours of both Abell 399 and Abell 401 (Fig. 3) are elongated along a N-N-E/S-S-W axis, which is the major direction of the pair. The Abell 401 brightness contours appear mildly disturbed with a centroid shift to the northeast. Consistent with the expectation of the $P 4 / P 0$ power ratios reported in Table 1 , they are less regular than the contours of Abell 1795, Abell 2029 and Abell 478. Abell 399 looks even more irregular; in particular a sharp edge can be observed to the southeast of the cluster core.

Using the same data set as used in this work, Sakelliou \& Ponman (2004) studied the gas brightness and temperature in specific angular sectors of both clusters and along the major direction of the pair. From this analysis, they conclude that, currently, the clusters are just starting to mildly interact and that the sub-features found in their inner regions are related to the individual merging histories of each cluster separately, rather than to the remnant of a previous merger of the two systems.

The temperature maps of Abell 399 and Abell 401 are reported in Fig. 3 to the same spatial scale. We notice a higher average temperature for Abell $401(k T \simeq 8 \mathrm{keV})$ than for Abell 399 $(k T \simeq 7 \mathrm{keV})$, consistent with the analysis of Sakelliou \& Ponman (2004). The temperature maps of both clusters appear as strongly irregular, without any elliptical symmetry or large scale correlation with gas brightness structure, contrary to what is observed for the relaxed clusters in our sample. Moreover, instead of cool cores, their central regions host some hot substructure embedded in the colder ICM. We further confirm the temperature increment described by Sakelliou \& Ponman (2004) to the south of Abell 401, in the direction of Abell 399. However, we do not detect any temperature increment toward the north of Abell 399 along the expected interaction axis of the cluster pair. More generally speaking, most of the temperature irregularities detected here do not have any morphological link with the interaction axis of the cluster pair, and have typical sizes more related to the central region of each cluster than to the overall cluster system.

\subsubsection{Abell 2065}

Abell 2065 has been previously studied by Markevitch et al. (1999) using ROSAT and ASCA data, and by Chatzikos et al. (2006) using Chandra data. These observations revealed the asymmetric morphology of this cluster characterised by a highly elongated inner region that seems to link together the two $\mathrm{cD}$ galaxies located in the cluster centre. The temperature structure is also highly asymmetric with a hotter region to the southeast. Furthermore, the Chandra observation revealed two cold cores coinciding with the cluster central $\mathrm{cD}$ galaxies. 
The XMM-Newton temperature map of Abell 2065 in Fig. 3 shows the presence of a hot $(k T \simeq 6.5 \mathrm{keV})$ bow-like region, from about 3 to 6 arcmin to the southeast of a cold cluster core $(k T \simeq 4.5 \mathrm{keV})$. This feature by itself is not isothermal but rather appears to embed an even hotter sub-feature $(k T \simeq 8.5 \mathrm{keV})$ to the eastern cluster outskirts. The map also shows a cold region $(k T \simeq 4 \mathrm{keV})$ located at about 7 arcmin to the northwest of the cluster core, appearing to be linked to this core by an extended tail.

Already reported in previous works (Markevitch et al. 1999; Chatzikos et al. 2006), the hot bow-like region is located next to an abrupt variation of gas surface brightness visible in Fig. 1. For this reason, it probably indicates the presence of a cold front, as discussed in Sect. 7.

\subsubsection{Abell 2256}

The X-ray image of Abell 2256 in Fig. 1 shows a complex structure with two X-ray peaks, one of which is coincident with the cluster central dominant galaxy, while the other is located at 2 arcmin to the northwest. This cluster has been observed with ROSAT and ASCA (e.g. Briel et al. 1991; Markevitch 1996) and more recently with Chandra. This latter observation revealed an even more irregular X-ray morphology, the presence of a third subgroup to the east, and a sharp edge to the southeast of the northwest peak that has been identified with a "cold front" (Sun et al. 2002).

The XMM-Newton temperature map of Abell 2256 in Fig. 3 shows a bimodal temperature structure along the cluster major elongation axes. Consistent with Sun et al. (2002), we find that the gas in the northwest peak, is the coldest of the cluster with a temperature of $k T \simeq 4.5 \mathrm{keV}$. Unlike previous work, our temperature map also shows a clear, hot $(k T \simeq 9 \mathrm{keV})$, bow-like region to the east. This hot region is located just outside an abrupt variation of the gas surface brightness, also clearly visible from the compression of the isocontour levels in Fig. 3. As shown in Sect. 7, this new feature is likely to be related to another cold front in the cluster ICM.

\section{Radial temperature structure and cluster mass}

X-ray emission from round relaxed clusters of galaxies is often used to estimate the cluster mass, assuming hydrostatic equilibrium and spherical symmetry. Using hydro $N$-body simulations, Rasia et al. (2006) investigated the accuracy of the mass estimate and found that, in the best of cases, there is at least a $10 \%$ discrepancy between the true and the estimated mass. They claimed that one of the reasons for such a discrepancy is related to small, non radial thermal substructure, which seems to be always present, even in the most relaxed systems produced by any hydro $N$-body simulation.

To test whether this problem may also be present in real clusters, we used our temperature maps and selected specific cluster sectors in which the gas temperature is either hotter or colder than the gas mean temperature. From these sectors, we extracted temperature and brightness profiles, then used these profiles to estimate the relative cluster mass profiles.

For this test we consider only two of the three more relaxed clusters in our cluster sample: Abell 2029 and Abell 1795. Abell 478, the third round cluster in our sample, was not considered for such a test because, as explained in Sect. 8, it is located in a particular sky region characterised by strong angular variations of the neutral hydrogen column density, $N_{\mathrm{h}}$, across the cluster field of view. If not treated properly, these strong $N_{\mathrm{h}}$ variations may introduce spurious thermal features that cannot be easily disentangled from the real ones.

\section{ICM surface brightness and temperature profiles}

Brightness and temperature profiles associated with hot and cold regions of temperature maps for Abell 2029 and Abell 1795 are shown in Fig. 4 with different colours.

The brightness profiles $\Sigma_{\mathrm{x}}(r)$ have been obtained by averaging the brightness estimator of Eq. (9) within logarithmically equispaced elliptical annuli of $\mathrm{N}$ pixels $\{k, l\}_{r}$, with normalisation by the exposure weighted instrument area $a(k, l)$ of Eq. (8):

$$
\begin{aligned}
\widehat{\Sigma_{\mathrm{X}}}(r) & =\frac{1}{N} \sum_{\left\{k, l_{r}\right.} \widehat{L_{\mathrm{X}}}(k, l), \\
& =\frac{1}{N} \sum_{\left\{k, l_{r}\right.} \frac{n_{\mathrm{evt}}(k, l)-n_{\mathrm{bck}}(k, l)}{a(k, l)} .
\end{aligned}
$$

We then estimated the brightness variance by considering uncertainties to be only related to the Poisson fluctuation of $n_{\mathrm{evt}}(k, l)$ :

$$
\widehat{\sigma_{\Sigma_{\mathrm{x}}}}(r) \simeq \frac{1}{N} \sqrt{\sum_{\left\{k, l_{\}_{r}}\right.} \frac{\widehat{\sigma_{n_{\mathrm{evt}}^{2}}}(k, l)}{a^{2}(k, l)}}=\frac{1}{N} \sqrt{\sum_{\{k, l\}_{r}} \frac{n_{\mathrm{evt}}(k, l)}{a^{2}(k, l)}}
$$

The spectroscopic temperature profiles $\widehat{T}_{\mathrm{x}}(r)$ have been estimated by fitting the spectral model $F_{\text {evt } k, l}\left(T, Z, N_{\mathrm{h}}, e\right)$ of Eq. (2) to the data associated with a set of logarithmically equispaced sector annuli. Given the high statistics available for each annulus, we were able to perform spectral estimations following a $\chi^{2}$ minimisation process, and get straightforward estimations of confidence intervals.

\section{Cluster mass profiles}

The surface brightness and temperature profiles extracted from the hottest and coldest sectors of A1795 and A2029 (see Fig. 4) have been used to estimate the cluster mass profile. To do so, we adopt the approach proposed by Vikhlinin et al. (2006), that consists in modelling the 3-d density and 3-d temperature profiles and fitting the projected quantities to the corresponding data set.

The 3-d gas density is modelled by a double and modified $\beta$ model with 9 parameters. Introducing $n_{\mathrm{p}}$ and $n_{\mathrm{e}}$, the proton and electronic density, respectively, we get:

$$
\begin{aligned}
{\left[n_{\mathrm{p}} n_{\mathrm{e}}\right](r)=} & n_{0}^{2} \frac{\left(r / r_{\mathrm{c}}\right)^{-\alpha}}{\left[1+\left(r / r_{\mathrm{c}}\right)^{2}\right]^{3 \beta-\alpha / 2}} \frac{1}{\left[1+\left(r / r_{\mathrm{s}}\right)^{3}\right]^{\epsilon / 3}} \\
& +\frac{n_{02}^{2}}{\left[1+\left(r / r_{\mathrm{c} 2}\right)^{2}\right]^{3 \beta_{2}}} .
\end{aligned}
$$

The projected surface brightness profile, $\Sigma(r)$, as observed within a given energy band $\Delta E$ (here $0.7-2.5 \mathrm{keV}$ ), is then obtained by integrating the ICM brightness, $\epsilon_{\mathrm{ICM}}(T)$, along the line of sight:

$\Sigma_{\mathrm{x}}(r)=\frac{1}{d^{2}(1+z)^{4}} \int \epsilon_{\mathrm{ICM}}\left[T\left(r_{1}\right)\right]\left[n_{\mathrm{p}} n_{\mathrm{e}}\right]\left(r_{1}\right) \mathrm{d} l$,

where $z$ is the redshift of the source located at distance $d$, where $r_{1}=\sqrt{r^{2}+l^{2}}$, and where $\epsilon_{\mathrm{ICM}}(T)$ can be modelled from the source radiation power of Eq. (3), so as to account for instrument response and effective area. Normalising with the same 

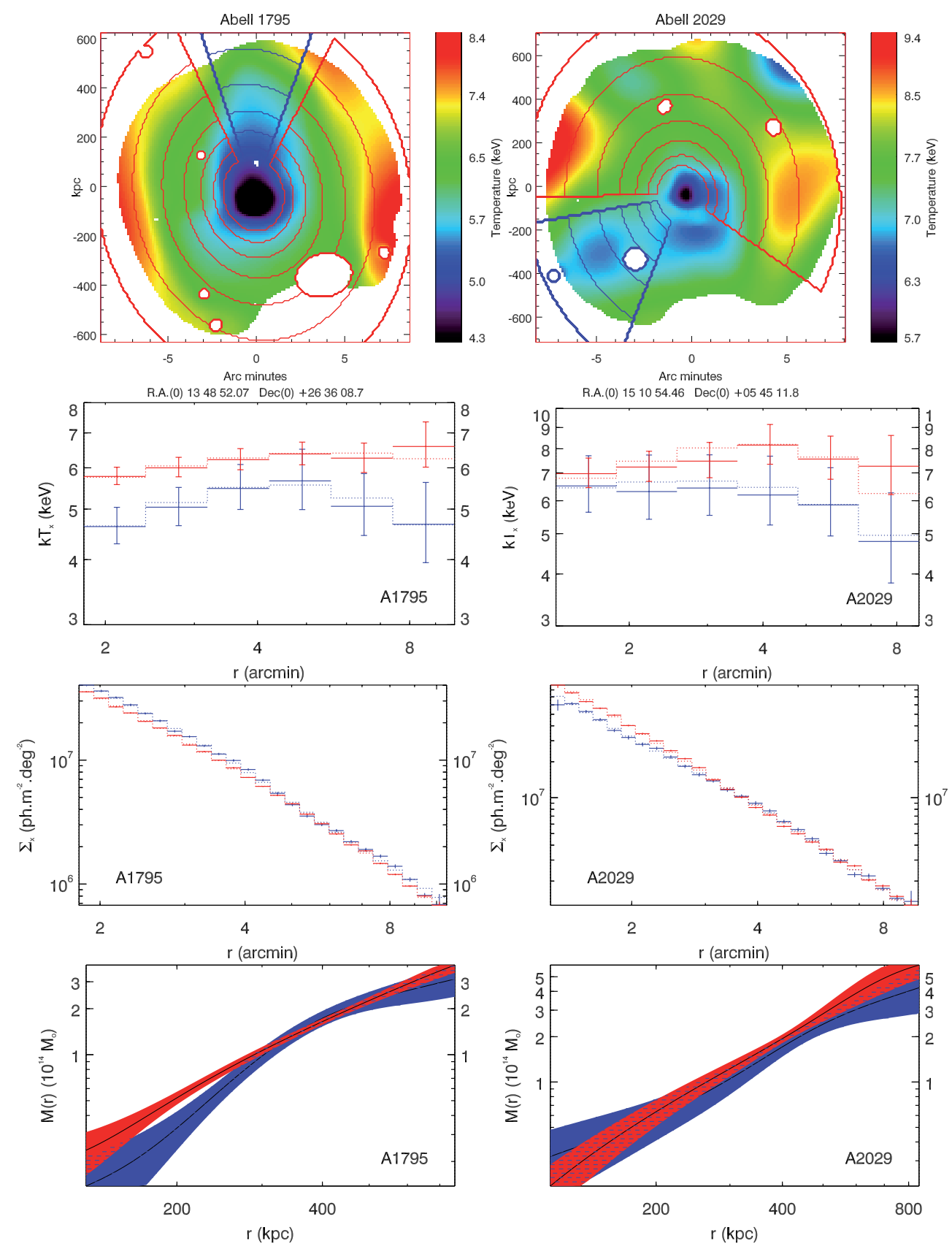

Fig. 4. Spectroscopic temperature (top), brightness (middle) and derived mass profiles (bottom) corresponding to the selected red and blue sectors of Abell 1795 (left) and Abell 2029 (right), respectively. Dashed lines on temperature and brightness profiles correspond to fits of the projected functions $\left\{T_{\mathrm{X}}(r), \Sigma_{\mathrm{X}}(r)\right\}$ used for deriving masses, see Eqs. (18) and (15). Error bars on temperature profiles are $68 \%$ confidence levels. Dispersions of brightness and mass profiles are distribution variances.

average quantities, $T_{\mathrm{o}}, Z_{\mathrm{o}}, N_{\mathrm{ho}}$, as used for computing the weighted instrument area in Eq. (8), we get:

$\epsilon_{\mathrm{ICM}}(T)=\frac{\int_{\Delta E} F_{\mathrm{ICM}}\left(T, Z, N_{\mathrm{h}}, e\right) \mathrm{d} e}{\int_{\Delta E} F_{\mathrm{ICM}}\left(T_{\mathrm{o}}, Z_{\mathrm{o}}, N_{\mathrm{ho}}, e\right) \mathrm{d} e}$.

The 3-d temperature profile is modelled by a 5-parameter broken power-law, with transition region:

$T(r)=T_{\mathrm{o}} \frac{\left(r / r_{\mathrm{t}}\right)^{-a}}{\left[1+\left(r / r_{\mathrm{t}}\right)^{b}\right]^{c / b}}$,

then integrated along the line of sight to get a projected profile of "spectroscopic-like" temperatures, as defined in Eqs. (14) and (15) of Mazzotta et al. (2004):

$T_{\mathrm{x}}(r)=\frac{1}{\int w\left(r_{1}\right) \mathrm{d} l} \int w\left(r_{1}\right) T\left(r_{1}\right) \mathrm{d} l$,

with weighting factor $w(l)=\frac{n^{2}(l)}{T^{3 / 4}(l)}$.
After fitting the projected ICM brightness $\Sigma_{\mathrm{x}}(r)$, and temperature profile $T_{\mathrm{S}}(r)$ to the observable set $\left\{\widehat{\Sigma_{\mathrm{x}}}(r), \widehat{T_{\mathrm{x}}}(r)\right\}$, and estimating the related 3 -d density $\rho(r)$, and temperature $T(r)$, we use hydrostatic equilibrium of the ICM to derive mass profiles (see e.g. Sarazin 1988):

$$
M(r)=-3.68 \times 10^{13} M_{\odot} T(r) r\left[\frac{\mathrm{d} \log \rho}{\mathrm{d} \log r}+\frac{\mathrm{d} \log T}{\mathrm{~d} \log r}\right] .
$$

Best fits of the projected ICM brightness and temperature profiles with associated cluster mass profiles $M(r)$, are shown in Fig. 4. The confidence intervals on mass profiles have been estimated by minimising the distance between the projected models and a set of random realisations of observed profiles. These profiles have been obtained assuming Gaussian statistics around observed values $\left\{\widehat{\Sigma_{\mathrm{x}}}(r), \widehat{T}_{\mathrm{x}}(r)\right\}$, with the constraint of rejecting realisations leading to the non-physical solution of nonmonotonically increasing mass profiles. 


\subsection{Abell 1795}

The Abell 1795 ICM temperature map reveals a cold region to the north of the cluster, while the gas temperature is observed as hotter elsewhere in the 2 to 5.5 arcmin range of cluster radii. We selected two complementary "cold" and "hot" cluster sectors in order to compute surface brightness and temperature profiles in Fig. 4. As we want to ignore effects of gas thermal variations near the cluster core, we excluded this region from our analysis and computed profiles within a radii range of $1.5-8$ arcmin. We further centred sectors so as to fit the cluster brightness isophotes at large radii, therefore the cool core appears as being shifted with regard to our sectors in Fig. 4.

Consistent with what is observed on the temperature map, we notice that the absolute temperatures in the two sectors are significantly different. Furthermore, we observe that the two temperature profiles show different shapes: the profile corresponding to the coldest sector peaks at approx 4 arcmin, while the complementary hottest profile is much flatter. The two complementary brightness profiles also show different shapes.

It is worth noticing that the resulting mass profiles show significantly different shapes (see Fig. 4). In particular, as for the temperature profiles, the mass profile of the coldest sector shows stronger gradient variation than the hottest one. These different shapes result in the observation that while the relative mass estimates are consistent with each other at large radii $(r>250 \mathrm{kpc})$ - and also consistent with previously published profiles of e.g. Ikebe et al. (2004) or Vikhlinin et al. (2006) - they become significantly different towards the innermost part of the cluster $(r<250 \mathrm{kpc})$.

We should conclude that, within the radii range investigated, the assumption of hydrostatic equilibrium for this cluster may be not valid.

\subsection{Abell 2029}

The Abell 2029 ICM temperature map reveals a non symmetric temperature structure within a region ranging from 2 to 5 arcmin cluster radii. We selected the coldest region to the southeast for computing a first set of brightness and temperature profiles, and a complementary hot sector for computing the second set.

As with Abell 1795, the discrepancy observed between temperature values on profiles in Fig. 4 is consistent with the radial thermal structure of the temperature map. In particular, the profile corresponding to the cold sector is almost flat $(k T \simeq$ $6.5 \mathrm{keV}$ ), while the profile corresponding to the hot sector has a positive gradient up to approx 4 arcmin radius (where it reaches $k T \simeq 8.5 \mathrm{keV}$ ), then decreases down to $k T \simeq 6 \mathrm{keV}$ at larger radii. It is important to say, however, that due to the limited statistics available for fitting temperatures in the coldest region of A2029, the temperature discrepancies we find are only marginal for most of the radial bins. However, as with A1795, we find that the surface brightness profiles of the two complementary sectors have different shapes.

Despite this, and in contrast with what we find for Abell 1795, the mass profiles derived for the two complementary cluster sector seem to have remarkably similar shapes that lead to a consistent mass estimate over the entire radial range considered $(r \in[200,800] \mathrm{kpc})$. This estimate is also consistent with previous works of e.g. Lewis et al. (2003) and Vikhlinin et al. (2006).

For this cluster, we can say that the temperature and surface brightness gradients compensate each other very well, leading to the same mass estimates regardless of the sector used. Thus, in this specific case, we may conclude that the hydrostatic equilibrium assumption is well satisfied.

\section{Cold fronts}

The temperature maps of both merging clusters Abell 2065 and Abell 2256 show two hot bow-like regions to the southeast of each cluster centre, which seem to be located next to abrupt variations of the gas surface brightness, as visible on photon maps in Fig. 1 or isocontour levels in Fig. 3. For this reason, they are likely to be related to cold fronts separating the dense and moving cluster cores from the hotter ICM of the cluster outskirts.

In order to investigate this hypothesis, for clusters Abell 2065 and Abell 2256 we extracted the ICM surface brightness and temperature profiles corresponding to sectors shown in Fig. 5. Located across the brightness front regions, these sectors follow the brightness isophotes and match regions with almost uniform isoradial thermal structure.

As expected from temperature and brightness maps, we clearly see two temperature jumps on the profiles located at a jump radius $r_{\mathrm{j}}$, also corresponding to a change of slope in surface brightness profiles. In order to check whether these features can be related to jumps in the 3 -d distributions of the gas density and temperature, we modelled these distributions by two disrupted functions.

The gas density profile is modelled by two independent $\beta$-models corresponding to regions located inside and outside the jump radius $r_{\mathrm{j}}$, respectively:

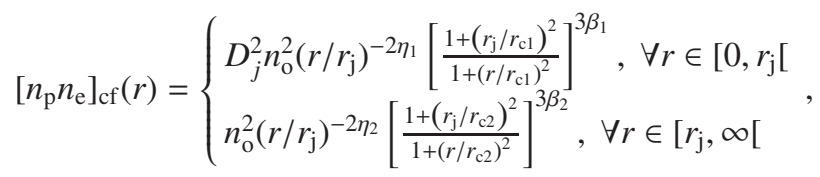

while the gas temperature is modelled by a step function:

$T_{\mathrm{cf}}(r)=\left\{\begin{array}{l}T_{\mathrm{o}}, \quad \forall r \in\left[0, r_{\mathrm{j}}[\right. \\ D_{T} T_{\mathrm{o}}, \quad \forall r \in\left[r_{\mathrm{j}}, \infty[.\right.\end{array}\right.$

The 3-d distributions $\left[n_{\mathrm{p}} n_{\mathrm{e}}\right]_{\mathrm{cf}}(r)$ and $T_{\mathrm{cf}}(r)$ are projected according to Eqs. (15) and (18) in order to derive X-ray brightness and "spectroscopic-like" temperature profiles, $\Sigma_{\mathrm{x}}(r)$ and $T_{\mathrm{x}}(r)$, which enables us to estimate all free parameters of Eqs. (20) and (21), including $r_{\mathrm{j}}$, by fitting the projected models to the data. Best fits of X-ray brightness and "spectroscopic-like" temperature profiles are shown in Fig. 5, with associated 3-d distributions of gas density and temperature. Similarly to the derivation of mass profiles in Sect. 6, the confidence intervals on 3-d profiles have been estimated by minimising the distance between the projected models and a set of random realisations of the observed profiles.

\subsection{Abell 2065}

The Abell 2065 sector is located within 5 arcminutes to the southeast of the cool elongated central cluster region visible in Fig. 1.

Fitting the disrupted density and temperature profile of Eqs. (20) and (21) for the southeastern sector of Abell 2065 leads to a jump radius of $r_{\mathrm{j}} \simeq 0.137_{-0.002}^{+0.002} \mathrm{Mpc}$, a density jump factor between regions located immediately above and below $r_{\mathrm{j}}$, of $D_{j} \simeq 1.29_{-0.01}^{+0.01}$, and a temperature jump factor of $D_{T} \simeq 1.49_{-0.14}^{+0.09}$. These values yield an almost continuous gas pressure across 

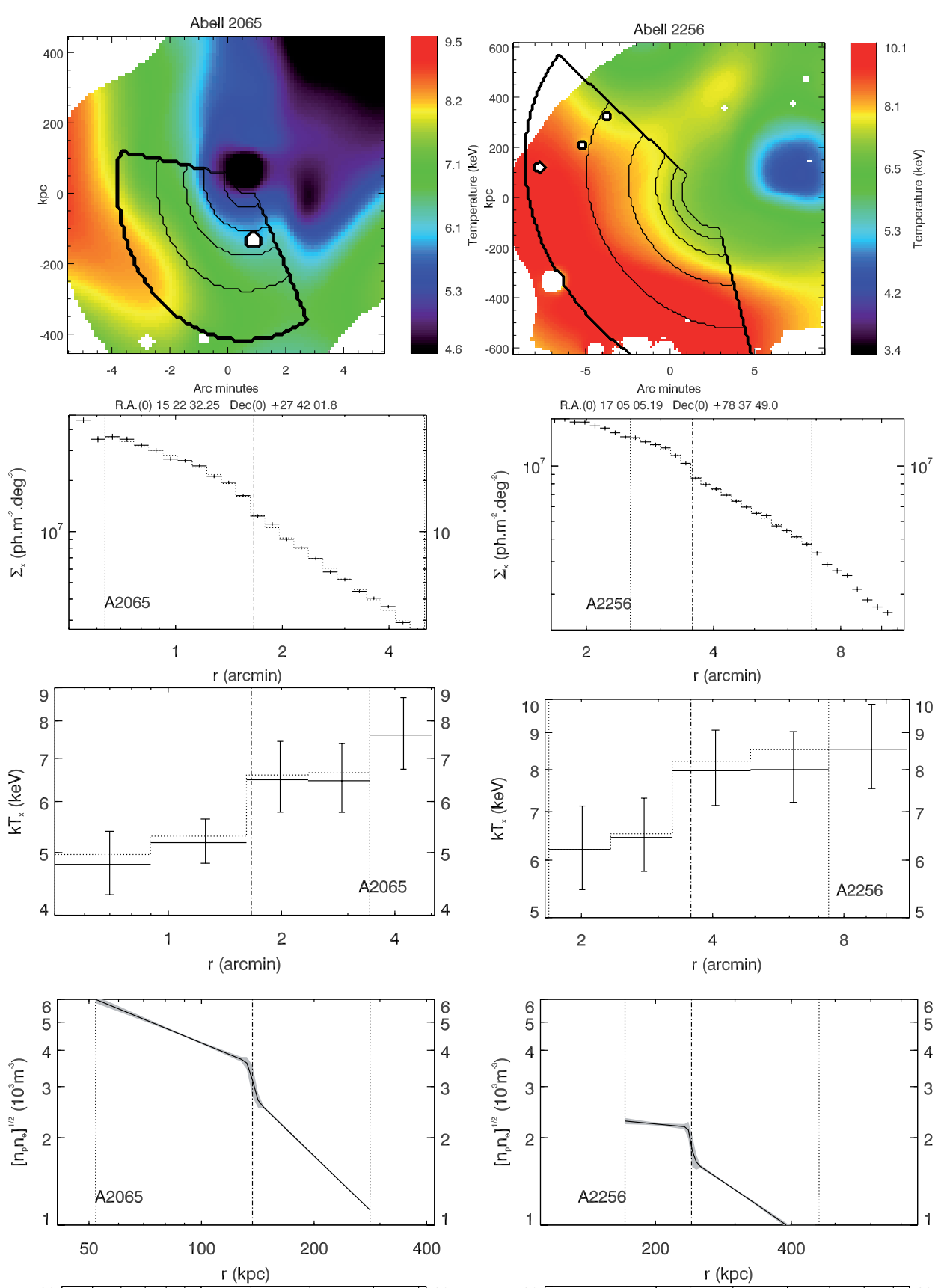

Fig. 5. From top to bottom: gas brightness, spectroscopic temperature, and derived density and temperature profiles corresponding to sectors shown on the top maps for Abell 2065 (left) and Abell 2256 (right), respectively. Dashed lines on temperature and brightness profiles corresponds to fits of the projected functions $\left\{T_{\mathrm{x}}(r), \Sigma_{\mathrm{x}}(r)\right\}$ (see Sect. 7). The front modelling and fitting region is bounded by vertical dashed lines on the gas brightness and spectroscopic temperature profiles, while the fitted front position $r_{\mathrm{j}}$ is reported by a vertical dot-dashed line. Error bars on the temperature profiles are $68 \%$ confidence levels. Dispersions reported on the $3 \mathrm{~d}$ profiles correspond to variances on each distribution.

the front. Interestingly, we notice that the value of $r_{\mathrm{j}}$ is consistent with the location of a density jump already reported by Chatzikos et al. (2006), who performed a sector analysis of the same cluster region using Chandra data. The additional detection of a temperature jump using XMM-Newton enables us to identify the feature as a cold front.

\subsection{Abell 2256}

The profiles shown in Fig. 5 correspond to a sector located within 8 arcmin to the southeast of the eastern cluster peak. As with Abell 2065, we detect a cold front feature on the profiles. The front is located at $r_{\mathrm{j}} \simeq 0.240_{-0.002}^{+0.002} \mathrm{Mpc}$ from the sector centre, with density and temperature jump factor of $D_{j}=1.30_{-0.02}^{+0.02}$ 

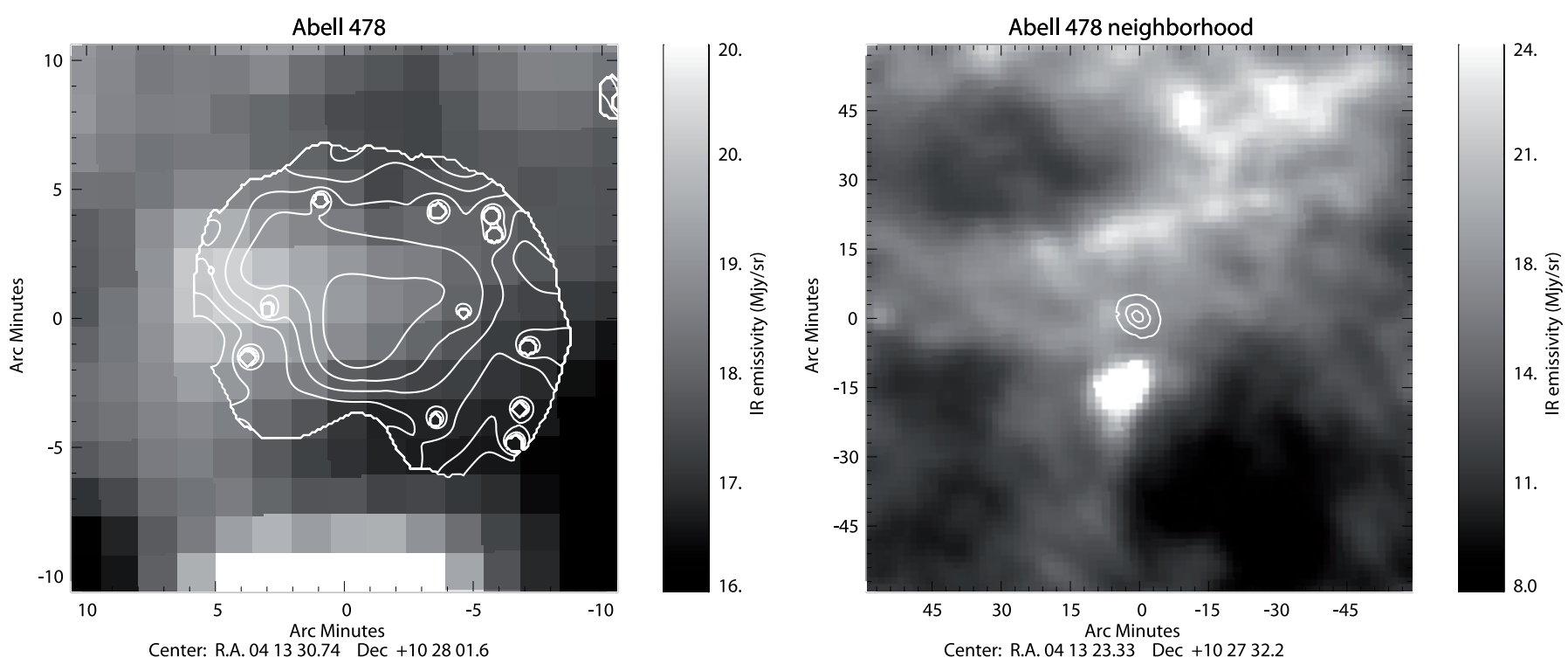

Fig. 6. Left image: IRAS/IRIS $100 \mu \mathrm{m}$ galactic dust emission map across the field of view of Abell 478 overlaid to the neutral hydrogen column density estimated by X-ray spectroscopy $\left(N_{\mathrm{h}}\right.$ isocontours are equispaced by $2 \times 10^{24} \mathrm{~m}^{-2}$ and decrease from a central value of $\left.30 \times 10^{24} \mathrm{~m}^{-2}\right)$. Right image: IRAS/IRIS $100 \mu \mathrm{m}$ galactic dust emission map of the 2 degree neighbourhood of A478, with A478 ICM brightness contours overlaid. The IR emissivity of the black region to the south of the cluster is of about $50 \mathrm{Mjy} / \mathrm{sr}$.

and $D_{T}=1.61_{-0.15}^{+0.19}$, respectively. Also here, the values of jump factors are consistent with continuous gas pressure across the front.

\subsection{Possible origin of the fronts}

As shown by Chatzikos et al. (2006) using Chandra data, the cold front feature seen in Abell 2065 is located to the southeast of a dual cluster core, which is likely the remnant of a binary interacting system, now merged. Following this scheme, the cold front may be related to the motion of the residual cool core of one of the former interacting clusters, with regard to the hot shocked and mixed gas of the present cluster.

In the same way, it is worth noticing that the front in Abell 2256 is located around a complex - at least dual - cluster core, as revealed by wavelet analyses of ROSAT PSPC (Slezak et al. 1994) and Chandra (Sun et al. 2002) images. Also in this case, we may infer that the cold front indicates the accretion of a subgroup, the core of which is now part of the eastern complex system in the cluster.

\section{Neutral hydrogen column density variations across the Abell 478 field of view}

The X-ray emission spectra of extragalactic sources are distorted by the neutral hydrogen absorption along the line of sight, whose origin is mostly galactic. We modelled this effect by introducing an absorption law which is a function of the neutral hydrogen column density, $N_{\mathrm{h}}$, to the ICM emission models $n_{\text {evt }}(k, l) F_{\text {evt }}\left(T, N_{\mathrm{h}},[e]\right)$, of Eq. (2). Depending on the knowledge of the average $N_{\mathrm{h}}$ value in the direction of the source observed, and on the spatial variation across the source field of view, this parameter may be fixed a priori or locally estimated from the observed ICM emission spectra. The average $N_{\mathrm{h}}$ across the field of view of each cluster in our sample was first estimated by fitting $N_{\mathrm{h}}$ absorbed emission models to the overall ICM emission spectra. For all clusters except Abell 478, these overall spectroscopic measurements were consistent with $N_{\mathrm{h}}$ values measured by Dickey \& Lockman (1990); for A478, the spectroscopic $N_{\mathrm{h}}$ was found to be about twice as high. Due to the inconsistency seen between both of these values, we left the $N_{\mathrm{h}}$ as a free parameter for X-ray spectroscopy. This $T-N_{\mathrm{h}}$ dual parameter spectralfitting process has enabled us to map the estimated $N_{\mathrm{h}}$ across the field of view of A478, using a similar wavelet algorithm to the ICM temperature mapping algorithm described in Sect. 4.1.2.

A map of the spatial distribution of $N_{\mathrm{h}}$ measured by X-ray spectroscopy across the field of view of A478 is shown by white isocontours on the left panel of Fig. 6. In order to reduce a possible spectroscopic bias with brightness gradient, the map has been obtained from a wavelet analysis limited to scales smaller than 2 arcmin. Significant wavelet coefficients have been selected according to a hard thresholding at $2 \times \sigma$. The $N_{\mathrm{h}}$ distribution appears irregular with an east-west elongation, an extended excess to the centre of the field of view, and a peak to the to the east. Starting from the central excess $\left(N_{\mathrm{h}} \simeq 30 \times 10^{24} \mathrm{~m}^{-2}\right)$, the $N_{\mathrm{h}}$ decreases strongly to the north and south of the field of view, up to values consistent with the average galactic value of Dickey \& Lockman $(1990)\left(N_{\mathrm{h}}<20 \times 10^{24} \mathrm{~m}^{-2}\right)$ at a distance of 5 arcmin from the centre.

The $N_{\mathrm{h}}$ central excess shown on the map of Fig. 6 has already been reported by Pointecouteau et al. (2004), who performed a spectroscopic analysis within sectors of the same data set as here. As already proposed by Pointecouteau et al. (2004), its origin can be investigated using the IRAS far-infrared survey of galactic dust emission at $100 \mu \mathrm{m}$, with angular resolution of about 4 arcmin. Indeed, the far-infrared galactic dust emission can be used as a tracer of the galactic neutral hydrogen column density, since dust emission is expected to be correlated to the galactic neutral hydrogen at high galactic latitude $(|b|>10 \mathrm{deg})$, as shown by e.g. Boulanger et al. (1996) and Schlegel et al. (1998). Notice however that this correlation is expected to be better constrained within low- $N_{\mathrm{h}}$ regions of the sky $\left(N_{\mathrm{h}}<5 \times 10^{24} \mathrm{~m}^{-2}\right)$ than within high- $N_{\mathrm{h}}$ regions like the Abell 478 neighbourhood, since the scatter of the IR/ $N_{\mathrm{h}}$ correlation is higher in such regions, possibly due to the presence of molecular hydrogen, as discussed by Boulanger et al. (1996). A 
galactic dust emission map at $100 \mu \mathrm{m}$ of the 2 degree neighbourhood of Abell 478 is shown in the right panel of Fig. 6. Coming from the last generation of IRAS maps obtained from the IRIS processing (Miville-Deschênes \& Lagache 2005), this map reveals the structure of the galactic filament where Abell 478 is located, to a resolution that cannot be reached by the radio survey of Dickey \& Lockman (1990), with angular resolution of 1 degree. The IR brightness of this map appears to be strongly structured in this sky region, with relative flux variations of more than $100 \%$. Within a 5 arcmin region centred on the brightness peak of A478, the IR emissivity is of about $18 \mathrm{Mjy} / \mathrm{sr}$. This value corresponds to a $N_{\mathrm{h}}$ density column of $33 \times 10^{24} \mathrm{~m}^{-2}$ following the IR/ $N_{\mathrm{h}}$ correlation factor of Boulanger et al. (1996). It is consistent with density column estimations obtained by X-ray spectroscopy, and encourages us to use the dust emission at $100 \mu \mathrm{m}$ as a tracer of the galactic neutral hydrogen distribution within this region of the sky.

We investigated the accuracy of this tracer by superimposing our $N_{\mathrm{h}}$ map to a portion of the IRAS map in the left panel of Fig. 6. Interestingly, we notice some spatial correlation between the IR and X-ray estimated $N_{\mathrm{h}}$ map. Indeed, both the central extended excess and the eastern peak shown on the $N_{\mathrm{h}}$ map correspond to similar structures in IR. However, both maps are not fully correlated in flux, since the maximum of the $N_{\mathrm{h}}$ map is located to the centre of the field of view, while the maximum of the IR map is located at the eastern peak. Observations of this sky region using infrared data of higher resolution may help to show conclusively whether the $N_{\mathrm{h}}$ excess detected near the centre of Abell 478 corresponds to a real feature, or if it is due to a bias of $\mathrm{X}$-ray spectroscopic estimations.

\section{Discussions and conclusions}

We present a multi-scale algorithm based on wavelets for mapping the ICM temperature structure within clusters of galaxies. Following the approach proposed by Bourdin et al. (2004), the basic scheme of this algorithm is i) estimate the searched parameter within square resolution elements, sampling the field of view at different scales, ii) filter the analysed signal in order to get wavelet coefficients, iii) threshold the wavelet transform obtained, in order to de-noise the signal and map its 2D structure. In order to perform a more regular reconstruction of structures and to reduce thresholding artifacts, we improved the algorithm so that, to filter the signal, we use a B2-spline wavelet instead of the Haar wavelet used in Bourdin et al. (2004) (see Sect. 4.1.2 for details). This algorithm was used to map the X-ray and temperature structure of a nearly complete X-ray flux limited cluster sample containing the eight clusters that, to date, have useful XMM-Newton observations. For self-consistency of the results, a similar thresholding approach was adopted for each target.

From previous work, three of the clusters in our sample namely A2029, A1795, and A478 - have been identified as relaxed systems, while the remaining clusters have been identified as major mergers. This classification is supported by the power ratios analysis of the surface brightness of these clusters, obtained from ROSAT observations (Buote \& Tsai 1996). The XMM-Newton photon images in Fig. 1 and the surface brightness contours in Fig. 3 confirm this previous classification: the $\mathrm{X}$-ray morphology of the relaxed systems is quite regular and elliptical symmetric while the morphology of the merging systems is more complex with the presence of multiple X-ray peaks.

In Fig. 3 we show the temperature maps for all clusters in our sample overlaid to the soft $(0.3-2.5 \mathrm{keV}) \mathrm{X}$-ray brightness isocontours. Even if with a very different degree of complexity, we notice that all clusters, including the most relaxed ones, show non radial thermal structure. Let us recall that, since for this work we are only interested in detecting highly significant thermal structure, we adopted a quite conservative approach which is based on the Donoho (1995) wavelet shrinkage. For this reason the angular resolution of the detected structure appears to be lower than the angular resolution expected from EPIC XMMNewton data. A wavelet shrinkage with constant threshold, as tested in Bourdin et al. (2004) on simulated observations, or used in Belsole et al. (2004, 2005) and Sauvageot et al. (2005) on real data, would reveal non radial thermal structure even on smaller angular scales.

Despite the relatively low angular resolution we find that, consistent with predictions from numerical hydro $N$-body simulations, the complexity of the thermal structure of clusters strongly depends on the dynamical status of clusters themselves. From Fig. 3 we can see that the temperature maps of the clusters in our sample may be classified into three different types, characterised by an increasing regularity of the thermal structure:

- “irregular", as for A399 and A401. While selected independently in our sample, these clusters actually form a binary and mildly interacting system. It has been proposed that the strong irregularity of their temperature structure is the result of previous merger activities within each cluster of the system, independently from the present interaction (Sakelliou \& Ponman 2004).

- "bimodal temperature", as for A2065 and A2256. These clusters are known to show some strong temperature anisotropies and to be presently overcoming a late stage of merging. They show an elongated geometry of surface brightness, with temperature maps characterised by a colder and hotter regions on opposite sides along the major direction of elongation. This thermal structure is dominated by the contrast between cool features to the one side, probably associated with accreted material, and a hotter region to the other side, possibly associated with shocked gas - see e.g. Markevitch et al. (1999) and Sun et al. (2002), for A2065 and A2256, respectively - and separated from the cluster core by a cold front;

- "regular", as for the relaxed clusters, A2029, A1795, and A478. These clusters have a characteristic cool core region surrounded by a hotter gas annulus region peaking at about $300-400 \mathrm{kpc}$ from the cluster centre. As with the surface brightness, the temperature map of these clusters is almost regular and elliptically symmetric, indicating a good relaxed status for these systems. It is worth noting, however, that beyond the overall elliptical symmetry, we also detect a number of significant non radial thermal structures outside their cores. This result seems to be consistent with what is predicted by hydro $N$-body simulations of cluster formation, where clusters continuously accrete small galaxy groups during and between major mergers, which lead to temperature irregularities near the region where groups are accreted.

To conclude, we would like to stress that, despite the large dispersion of intrinsic brightness and exposure times of the clusters in our sample, the multi-scale algorithm presented here enabled us to reveal the thermal structure of the ICM of each cluster. Apart the detection of strong features mainly present in merging systems - e.g. cold fronts -, we were also able to detect some mild non-radial temperature structure outside the core of relaxed clusters. We notice that the relative temperature variation associated with these irregularities at fixed radii is about $10 \%$. We 
show that these non radial thermal variations affect the measured radial temperature profiles (see Sect. 6), and lead, at least for one of our clusters - A1795 -, to an inconsistency of mass derivations obtained using hydrostatic equilibrium assumption. Nevertheless, for another cluster, A2029, the hydrostatic equilibrium assumption appears to be valid despite the presence of temperature irregularities.

These results enlighten the possibility of constraining the thermalisation status of the ICM and the departure from gas hydrostatic equilibrium within bright clusters, using data obtained with current X-ray observatories. It invites us to further investigations of ICM temperature anisotropies within larger samples of relaxed clusters - possibly at even larger cluster radii than here by assuming specifically an elliptical geometry of the wavelet analysis - and to a more accurate evaluation of the dispersions implied by these anisotropies on cluster mass estimations.

Acknowledgements. We thank Albert Bijaoui and Eric Slezak for their contribution to the conception of the wavelet imaging and spectral-mapping algorithms, and Jean-Luc Sauvageot for his help in reducing XMM-Newton data. We further thank the anonymous referee for suggestions and comments that improved the paper significantly. H.B. acknowledges the financial support from contract ASI-INAF I/023/05/0. This work is based on observations obtained with XMMNewton, and ESA science mission funded by ESA Member States and the USA (NASA).

\section{Appendix A: Background modelling for the EPIC cameras on board of the XMM-Newton satellite}

A typical list of photon impact detection provided by the EPIC CCD cameras gathers events associated with various processes that have to be taken into account for modelling the observed spectra of extended sources. In the case of ICM observations, the detected photons are at least related to two extended contributions: the observed source itself and the cosmic Xray background $(\mathrm{CXB})$. Furthermore, a significant fraction of the events-list is actually associated with false detections due to cosmic-ray induced particles interacting with the detector. Eventually, a known fraction of events is registered during readout periods of detectors, which leads to an additive noise due to wrong position registration of these so-called "out-of-time" events.

We model, as an overall "background contribution" to the event spectrum $n_{\text {evt }}(k, l) F_{\text {evt }}\left(T, Z, N_{\mathrm{h}}, e\right)$ of Eq. (2), a combination of normalised spectral contributions associated with $\mathrm{CXB}$, $F_{\mathrm{CXB}}(e)$, induced particles, $F_{\mathrm{p}}(e)$, and readout noise, $F_{\text {oot }}(k, l, e)$ :

$$
\begin{aligned}
n_{\mathrm{bck}} F_{\mathrm{bck}}(k, l, e)= & E(k, l, e) \times n_{\mathrm{CXB}} F_{\mathrm{CXB}}(e) \\
& +n_{\mathrm{oot}}(k, l) F_{\mathrm{oot}}(k, l, e)+n_{\mathrm{p}} F_{\mathrm{p}}(e),
\end{aligned}
$$

where $n_{\mathrm{CXB}}, n_{\mathrm{p}}$ and $n_{\mathrm{oot}}$ are normalisation terms, and where $F_{\mathrm{CXB}}(e)$ is corrected for spatially variable exposure $E(k, l, e)$ (see Eq. (1)), as it is related to a physical observation. The CXB spectrum $F_{\mathrm{CXB}}(e)$ is modelled as the combination of a soft radiation associated with foreground galactic gas and a broad band contribution accounting for extragalactic background of unresolved AGNs. Following Lumb et al. (2002) and Kuntz \& Snowden (2000), we use a two temperature thermal radiation $(k T 1=0.074 \mathrm{keV}, k T 2=0.204 \mathrm{keV})$ and a power-law $(\gamma=1.42)$ for modelling the galactic and extragalactic contributions, respectively. The cosmic-ray induced particle background $F_{\mathrm{p}}(e)$ has been modelled from cumulated expositions of telescopes to the particles, during in-flight calibration phases.
The readout noise contribution $F_{\text {oot }}(k, l, e)$ is estimated by integrating and normalising the overall signal along CCD columns. While normalisation of the readout noise is known a priori for a given observing mode, normalisations of both the CXB spectrum and particle background must be set by fitting our background model, $n_{\text {bck }} F_{\text {bck }}(e)$, to an observed "background spectrum". To extract this spectrum, we select events from an external ring of the field-of-view with radius range of 10.5-12 arcmin, which is a region where background emissivity is already dominant but where effective area uncertainties are lower than they would be for even larger radii.

\section{References}

Arnaud, M., Neumann, D. M., Aghanim, N., et al. 2001, A\&A, 365, L80 Balucinska-Church, M., \& McCammon, D. 1992, ApJ, 400, 699

Bauer, F. E., Fabian, A. C., Sanders, J. S., Allen, S. W., \& Johnstone, R. M. 2005, MNRAS, 359, 1481

Belsole, E., Pratt, G. W., Sauvageot, J.-L., \& Bourdin, H. 2004, A\&A, 415, 821 Belsole, E., Sauvageot, J.-L., Pratt, G. W., \& Bourdin, H. 2005, A\&A, 430, 385 Bijaoui, A., \& Jammal, G. 2001, Signal Processing, 81, 1789

Bijaoui, A., \& Rue, F. 1995, Signal Processing, 46, 345

Boulanger, F., Abergel, A., Bernard, J.-P., et al. 1996, A\&A, 312, 256

Bourdin, H., Sauvageot, J.-L., Slezak, E., Bijaoui, A., \& Teyssier, R. 2004, A\&A, 414,429

Briel, U. G., Henry, J. P., Schwarz, R. A., et al. 1991, A\&A, 246, L10

Buote, D. A., \& Tsai, J. C. 1996, ApJ, 458, 27

Cappellari, M., \& Copin, Y. 2003, MNRAS, 342, 345

Chatzikos, M., Sarazin, C. L., \& Kempner, J. C. 2006, ApJ, 643, 751

Clarke, T. E., Blanton, E. L., \& Sarazin, C. L. 2004, ApJ, 616, 178

Coifman, R., \& Donoho, D. L. 1995, in Lecture Notes in Statistics: Wavelets and Statistics (Springer-Verlag), 103125

Curry, H. B., \& Schoenberg, I. J. 1947, Bull. Amer. Math. Soc., 53, 1114

Dickey, J. M., \& Lockman, F. J. 1990, ARA\&A, 28, 215

Donoho, D. L. 1995, IEEE Transactions on Information Theory, 41, 613

Ebeling, H., Edge, A. C., Bohringer, H., et al. 1998, MNRAS, 301, 881

Ettori, S., Fabian, A. C., Allen, S. W., \& Johnstone, R. M. 2002, MNRAS, 331, 635

Forman, W., \& Jones, C. 1982, ARA\&A, 20, 547

Grevesse, N., \& Sauval, A. J. 1998, Space Sci. Rev., 85, 161

Ikebe, Y., Böhringer, H., \& Kitayama, T. 2004, ApJ, 611, 175

Kuntz, K. D., \& Snowden, S. L. 2000, ApJ, 543, 195

Lewis, A. D., Buote, D. A., \& Stocke, J. T. 2003, ApJ, 586, 135

Lumb, D. H., Warwick, R. S., Page, M., \& De Luca, A. 2002, A\&A, 389, 93

Mallat, S. 1998, A wavelet tour of signal processing (Academic Press)

Markevitch, M. 1996, ApJ, 465, L1

Markevitch, M., Sarazin, C. L., \& Vikhlinin, A. 1999, ApJ, 521, 526

Markevitch, M., Ponman, T. J., Nulsen, P. E. J., et al. 2000, ApJ, 541, 542

Markevitch, M., Vikhlinin, A., \& Mazzotta, P. 2001, ApJ, 562, L153

Mazzotta, P., Rasia, E., Moscardini, L., \& Tormen, G. 2004, MNRAS, 354, 10

Miville-Deschênes, M.-A., \& Lagache, G. 2005, ApJS, 157, 302

Nevalainen, J., Markevitch, M., \& Lumb, D. 2005, ApJ, 629, 172

Pointecouteau, E., Arnaud, M., Kaastra, J., \& de Plaa, J. 2004, A\&A, 423, 33

Rasia, E., Ettori, S., Moscardini, L., et al. 2006, MNRAS, in press

Sakelliou, I., \& Ponman, T. J. 2004, MNRAS, 351, 1439

Sanders, J. S. 2006, MNRAS, 371, 829

Sanders, J. S., \& Fabian, A. C. 2001, MNRAS, 325, 178

Sanderson, A. J. R., Finoguenov, A., \& Mohr, J. J. 2005, ApJ, 630, 191

Sarazin, C. L. 1988, X-ray emission from clusters of galaxies Cambridge

Astrophysics Series (Cambridge: Cambridge University Press)

Sauvageot, J. L., Belsole, E., \& Pratt, G. W. 2005, A\&A, 444, 673

Schlegel, D. J., Finkbeiner, D. P., \& Davis, M. 1998, ApJ, 500, 525

Schuecker, P., Böhringer, H., Reiprich, T. H., \& Feretti, L. 2001, A\&A, 378, 408

Slezak, E., Durret, F., \& Gerbal, D. 1994, AJ, 108, 1996

Smith, R. K., Brickhouse, N. S., Liedahl, D. A., \& Raymond, J. C. 2001, ApJ, 556, L91

Sun, M., Murray, S. S., Markevitch, M., \& Vikhlinin, A. 2002, ApJ, 565, 867

Sun, M., Jones, C., Murray, S. S., et al. 2003, ApJ, 587, 619

Tamura, T., Kaastra, J. S., Peterson, J. R., et al. 2001, A\&A, 365, L87

Vikhlinin, A., Markevitch, M., Murray, S. S., et al. 2005, ApJ, 628, 655

Vikhlinin, A., Kravtsov, A., Forman, W., et al. 2006, ApJ, 640, 691 